\title{
Black coatings BSDF database
}

\section{Thijs Arts, Dana Tomuta, Volker Kirschner}

Thijs Arts, Dana Tomuta, Volker Kirschner, "Black coatings BSDF database," Proc. SPIE 11852, International Conference on Space Optics — ICSO 2020, 1185261 (11 June 2021); doi: 10.1117/12.2600049

SPIE Event: International Conference on Space Optics - ICSO 2021, 2021, Online SPIE. Only 


\section{International Conference on Space Optics-ICSO 2020}

Virtual Conference

30 March-2 April 2021

Edited by Bruno Cugny, Zoran Sodnik, and Nikos Karafolas
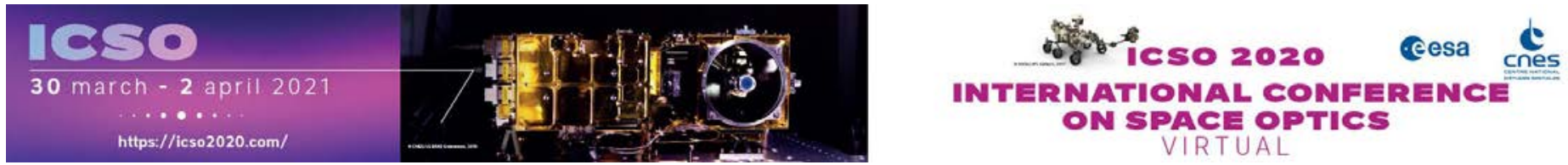

\section{Black coatings BSDF database}

\section{Cesa kos nacatang ecnes}




\title{
Black Coatings BSDF Database
}

\author{
Thijs Arts ${ }^{1}$, Dana Tomuta ${ }^{2}$, Volker Kirschner ${ }^{3}$ \\ European Space Research and Technology Centre (ESTEC), Keplerlaan 1, 2200AG \\ Noordwijk, The Netherlands
}

\begin{abstract}
Straylight requirements for optical instruments become ever more stringent. This applies not only to optical instruments in space, but also extends to optical ground support equipment (OGSE) and any other instrument that is sensitive to straylight. One of the means to control the straylight within instruments and OGSE's is the application of black coatings, e.g. on optical mounts, lens barrels and baffles. There are different types of black coatings on the market. The choice of a suitable black coating does not only depend on its optical properties, but also on the compatibility with the substrate material and its geometry. For many coatings, the manufacturers provide only a hemispherical reflectivity value, which is not very suitable for straylight predictions, as it does not include the angle dependent scattering behavior. This information is provided by the Bi-directional Scatter Distribution Function (BSDF). The optics and opto-electronics laboratories (OOEL) at ESTEC receive many requests to characterize black coatings by measuring the BSDF. The OOEL has created a database for black coatings to collect the BSDF data in support to space industry to facilitate the selection of the most suitable black coating and make more accurate predictions of straylight at instrument level. The database, called 'Black Coatings BSDF Database', will be presented here to showcase its functionalities. We will present some examples of BSDF data to demonstrate the added value over hemispherical reflectivity.
\end{abstract}

The BSDF data contained within the Black Coatings BSDF Database is structured according to coating type, measured wavelength and, if that information was available, according to specific information on the coating and/or substrate. So far, all BSDF measurements data has been measured at the OOEL using the two currently available CASI and Albatross TT scatterometers. Additional data will be added in the future. The European space industry is invited to contribute to the Black Coatings BSDF Database by either providing a sample to be characterized or contribute with already measured BSDF data. The Black Coatings BSDF database will be a database created for and by the European space industry. Access to the database is free and can be requested by contacting the OOEL via: ooel@esa.int.

Keywords: Black coatings, BSDF, Database, Straylight, Scattering

\section{INTRODUCTION}

Accuracy requirements for optical instruments become ever more stringent. As a result, straylight requirements on these instruments as well as their optical ground support equipment (OGSE) become ever more stringent to reach the required measurement accuracies. This applies not only to space based instruments, e.g. for Earth observation or astronomy, but also to ground based telescopes. One of the most commonly applied means to control straylight within these instruments and OGSE's is the application of black coatings, e.g. on optical

\footnotetext{
${ }^{1}$ (ATG Europe BV for ESA, email: mathijs.arts@esa.int)

2 (ESA, email: dana.tomuta@esa.int)

3 (ESA, email: volker.kirschner@esa.int)
} 
mounts, lens barrels and baffles. There are many black coatings available on the market, each with their own properties. Choosing a suitable black coating not only depends on its optical properties, but also on the compatibility with the substrate material and its geometry. Straylight simulations are usually performed to assess the effect of a black coating on the straylight performance of an instrument or OGSE. For many coatings, the manufacturers provide only a hemispherical reflectivity value, which is not very suitable for these straylight predictions, as it does not include the angle dependent scattering behavior. This information is provided by the Bi-directional Scatter Distribution Function (BSDF). As a result, the optics and opto-electronics laboratory (OOEL) at ESTEC receives many requests to measure the BSDF of black coatings. A database has been created to collect the BSDF of black coatings to support the space industry in their efforts to select the most suitable black coating and make more accurate predictions of straylight at instrument level. This database is called 'Black Coatings BSDF Database'.

The goal of this paper is to introduce the Black Coatings BSDF Database to the space community. First, the background of BSDF measurements is explained. Then some examples will be given to show the added benefit of using the BSDF as opposed to using only the hemispherical reflectivity. The final part of this paper will explain the functionalities of the database.

\section{BSDF MEASUREMENTS}

The Bidirectional Scattering Distribution Function (BSDF) describes the directional dependence of the reflected or transmitted optical energy. The BSDF is a fundamental optical property and describes the energy scattered into the reflective (BRDF) and transmissive (BTDF) hemisphere of a scattering surface as a function of the angle of the incident radiation $\left(\theta_{\mathrm{i}}\right)$ and the scatter angle $\left(\theta_{\mathrm{s}}\right)$. BSDF has been strictly defined as the ratio of the sample differential radiance to the differential irradiance under the assumption of a collimated beam. Power $\mathrm{P}$ in Watt (W) is used instead of intensity I in $\mathrm{W} / \mathrm{m}^{2}$, the value $\theta_{\mathrm{S}}$ is the polar angle in the scatter direction measured from surface normal and $\Omega$ is the differential solid angle (measured in steradians (sr)) through which $\mathrm{dP}_{\mathrm{S}}(\mathrm{W})$ scatters when $\mathrm{P}_{\mathrm{i}}(\mathrm{W})$ is incident on the surface. The cosine comes from the definition of radiance and may be viewed as a correction from the actual size of the scatter source to the apparent size (projected area) as the viewer rotates away from the surface normal [1]. A definition of the BSDF is given below.

$$
B S D F=\frac{\text { differential radiance }}{\text { differential irradiance }}=\frac{d P_{s} / d \Omega_{s}}{P_{i} \cos \theta_{i}}
$$

\section{Scattering Definitions}

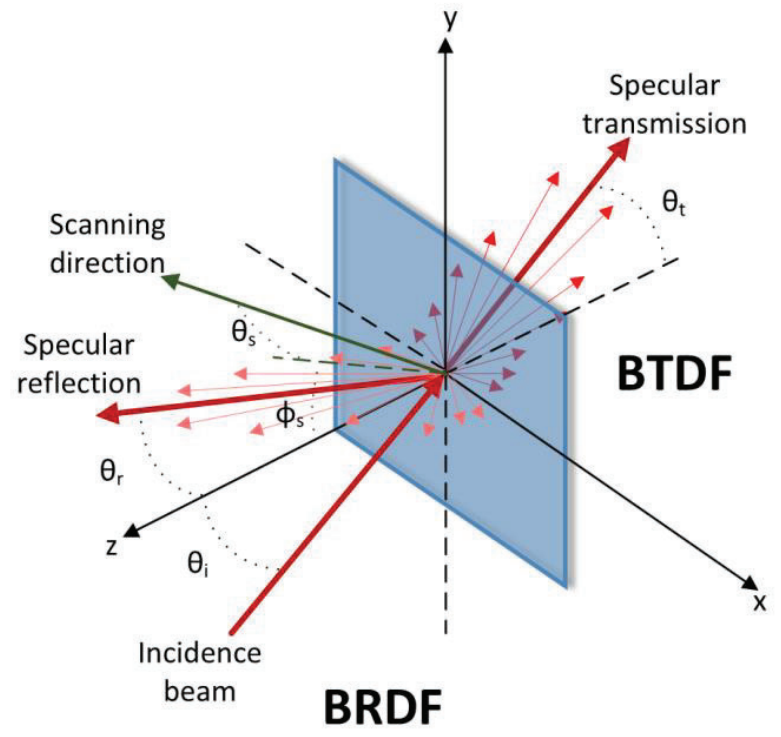

Figure 1: Illustration of the BSDF definition 
The BSDF is measured using a scatterometer. The general working principle of a scatterometer is: A monochromatic light source of power $\mathrm{P}_{\mathrm{i}}$, usually a laser, illuminates a spot on the sample to be measured under incidence angle $\theta_{\mathrm{i}}$. The sample is placed exactly in the centre of rotation of a detector arm that rotates around the sample to measure the signal $\mathrm{P}_{\mathrm{s}}$ for each angular position $\theta_{\mathrm{s}}$. The detector on the arm has a well-defined solid angle $\Omega_{\mathrm{s}}$ towards the sample.

At the moment of writing, two different scatterometers are available at the OOEL, being the CASI scatterometer from SMS [2] and the Albatross TT from Fraunhofer IOF [3]. The data presented in this paper has been measured using one of these scatterometers.

\section{BSDF IN COMPARISON TO HEMISPHERICAL REFLECTIVITY}

The hemispherical reflectivity is defined as the ratio between the reflected energy in the reflection hemisphere and the incident energy. This property is thus obtained by integrating all the reflected energy in the reflection hemisphere. Hemispherical reflectivity measurements are usually performed using an integrating sphere setup that allows only for a single fixed incidence angle. If one would perform a straylight analysis of an optical system and only the hemispherical reflectivity is known for the surfaces in this system, assumptions need to be made on the angular distribution of the reflected energy. Often it is assumed for straylight analysis that surfaces with a black coating have a near Lambertian behaviour that does not vary with incidence angle. These assumptions are, however, not correct. Especially towards larger incidence angles, many black coatings start to show a more specular behaviour and cannot be considered to be Lambertian. To illustrate this behaviour, some examples are given here.

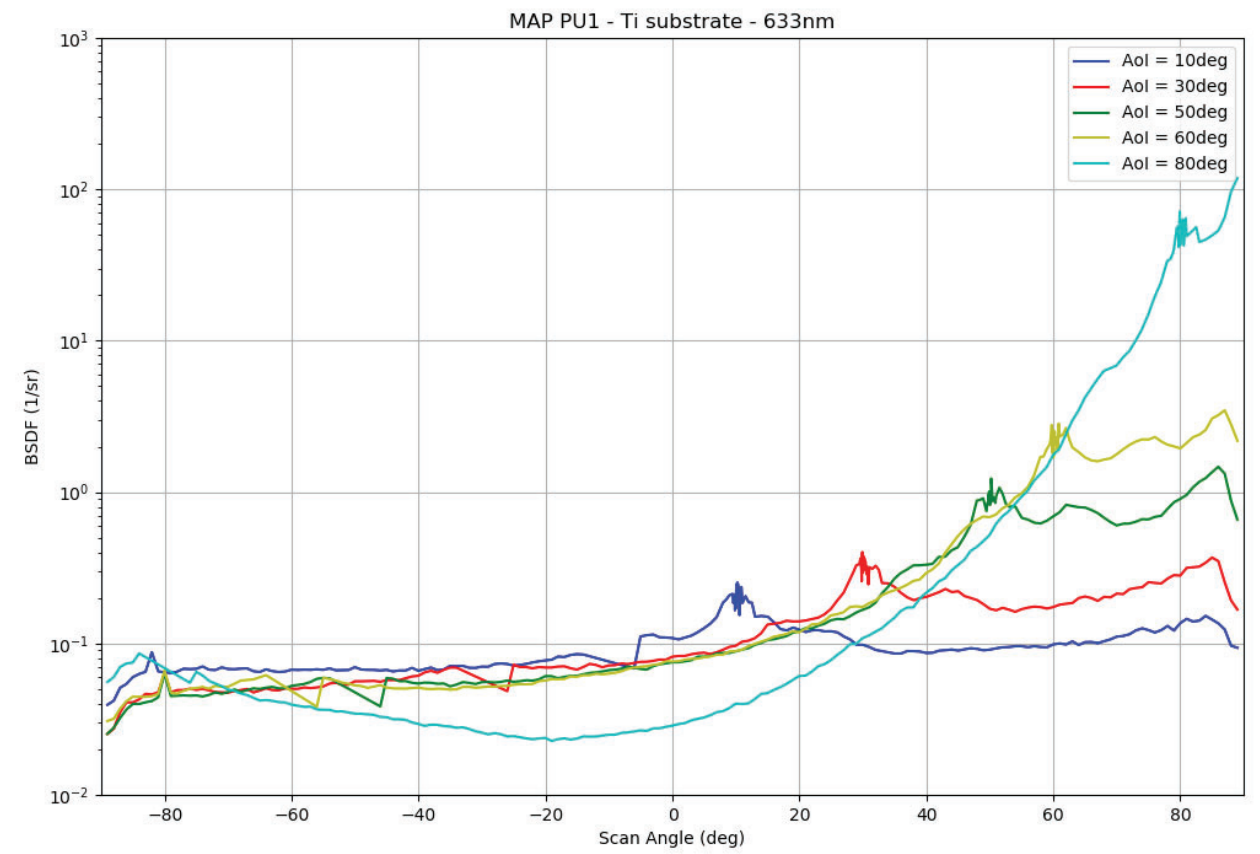

Figure 2: BSDF of the PU1 coating measured at a wavelength of $633 \mathrm{~nm}$ at several different incidence angles. For these measurements, the PU1 coating was applied to a titanium substrate. The measurements were performed using the CASI at the OOEL.

A good example is the BSDF at $633 \mathrm{~nm}$ of the black coating PU1, manufactured by the French company MAP Coatings, which is provided in Figure 2. The hemispherical reflectivity of PU1 coating has been measured and reported by the manufacturer to be slightly below 0.04 (at $633 \mathrm{~nm}$ ) [4]. At $10^{\circ}$ incidence angle the BSDF of PU1 shows a diffusive behaviour and only a small, broad specular peak can be observed. This specular peak becomes 
ever larger with increasing incidence angles. At the grazing angle of $80^{\circ}$ the coating behaves almost "mirrorlike" as the BSDF of the specular reflected peak is 2 orders of magnitude larger than the peak at $10^{\circ}$ incidence angle and the dynamic range of the BSDF is even close to 4 orders of magnitude. In addition, for all angles, the BSDF of PU1 is increasing towards larger scatter angles. This effect seems also to be increasing with increasing incidence angle.

Another example is the SolarBlack coating manufactured by the Irish company ENBIO. The BSDF of this coating at $532 \mathrm{~nm}$ is shown in Figure 3. The hemispherical reflectivity of SolarBlack given by the manufacturer seems to be around 0.05 [5]. The SolarBlack coating shows a more diffusive behaviour than the PU1 coating. However, just as the PU1 coating, SolarBlack becomes highly specular towards grazing angles.

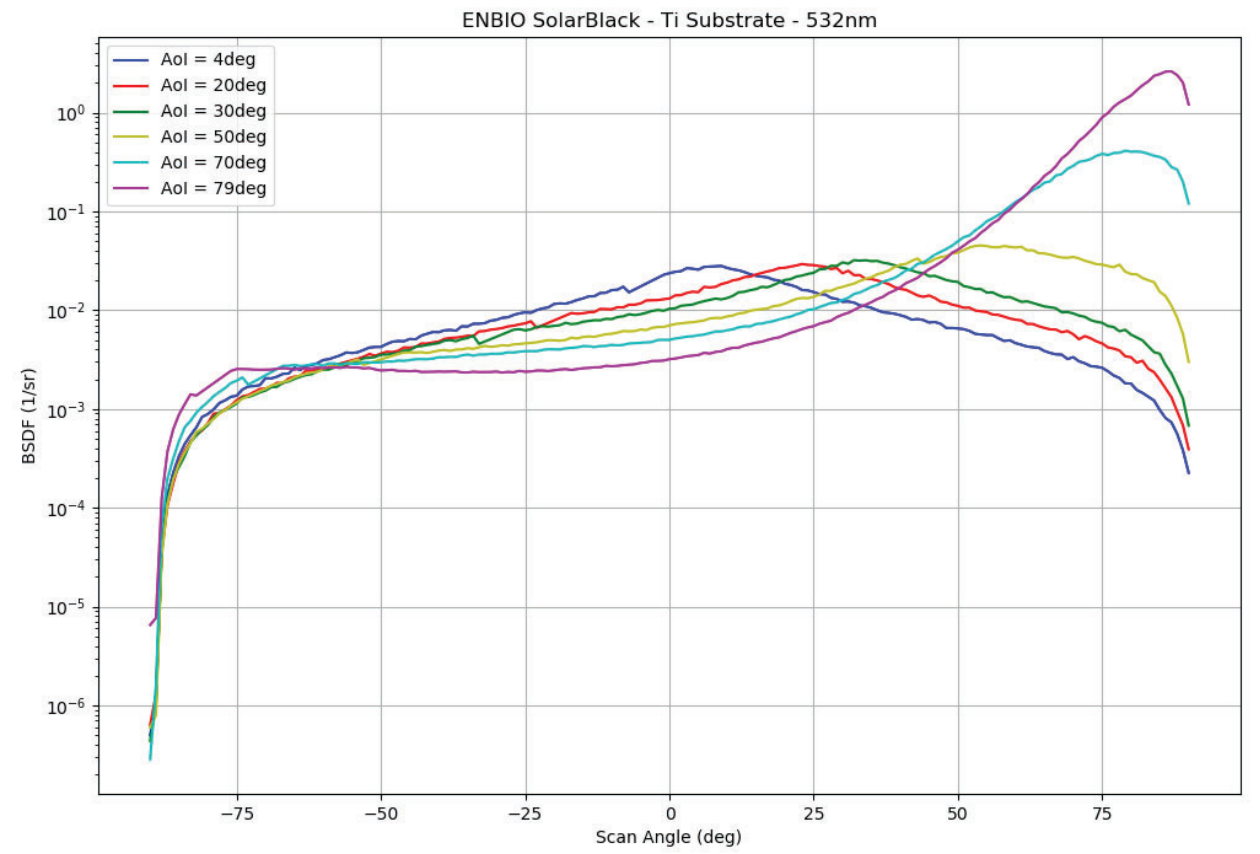

Figure 3: BSDF of the ENBIO SolarBlack coating at a wavelength of $532 \mathrm{~nm}$ at several different incidence angles. For these measurements, the SolarBlack coating was applied to a titanium substrate. The measurement setup used was the CASI at the OOEL.

Black coatings can also show a high anisotropic behaviour. This can for instance be seen for the BSDF that was measured on a foil of Acktar Nanoblack (Israelian company), which was measured in the frame of the ESAESO cooperation, both at the wavelength of 532nm (see Figure 4) and 2370nm (see Figure 5). 


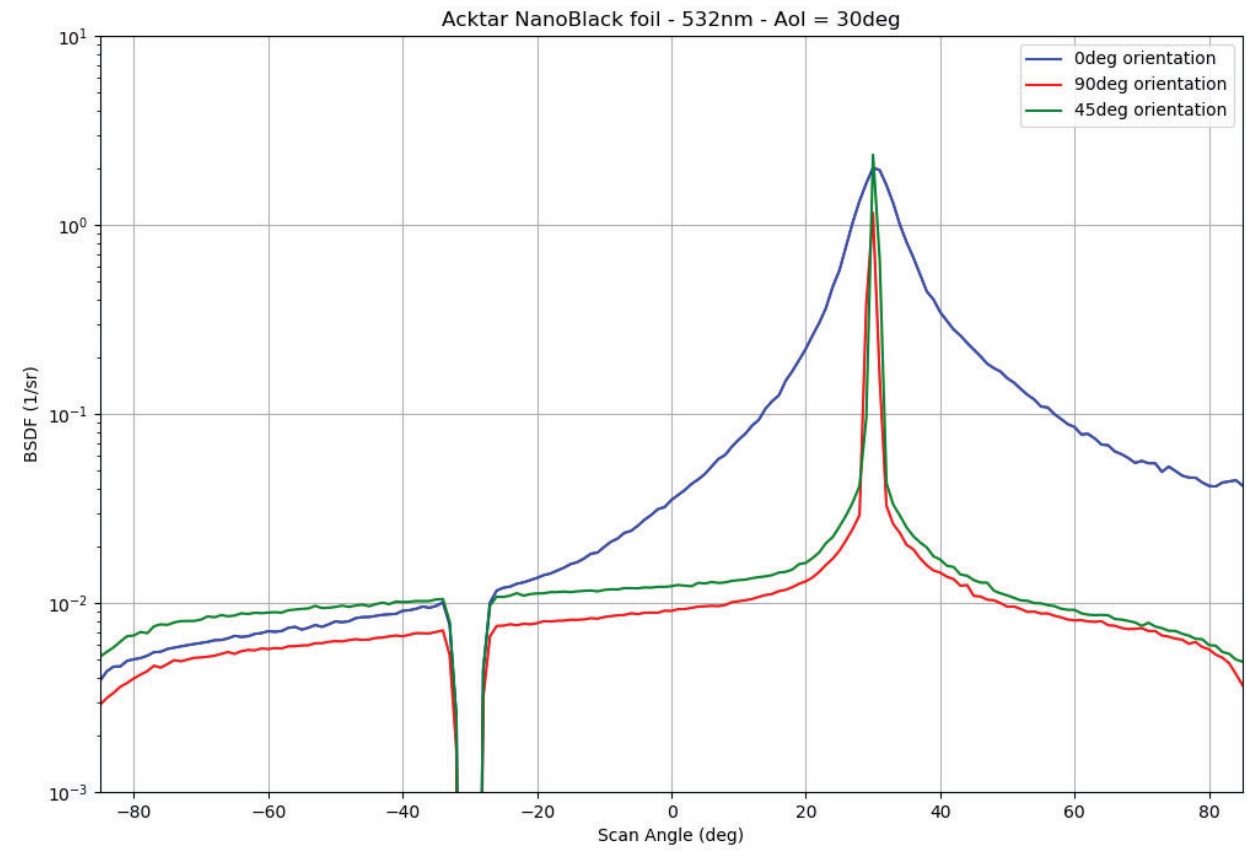

Figure 4: BSDF of Acktar NanoBlack foil at 532nm measured at 30deg incidence angle for different orientations of the sample. The measurement setup used for this was the Albatross TT at the OOEL. The test sample was measured in the frame of an ESA-ESO cooperation.

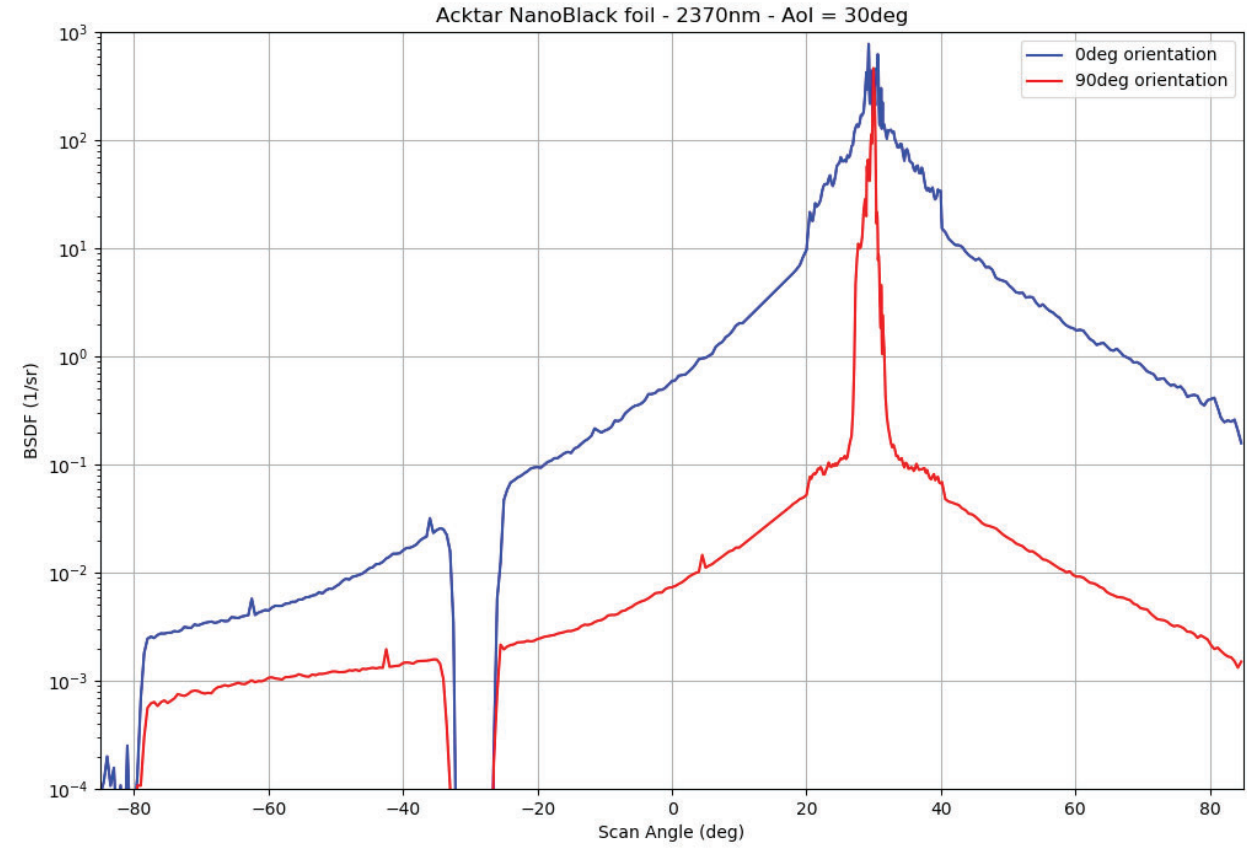

Figure 5: BSDF of Acktar NanoBlack foil at 2370nm measured at 30deg incidence angle for different orientations of the sample. The measurement setup used for this was the CASI at the OOEL. The test sample was measured in the frame of an ESA-ESO cooperation. 


\section{LAYOUT}

The Black Coatings BSDF Database can be accessed via the following link: https://tec.esa.int/sites/appZszq7G/SitePages/Home.aspx. To be granted access please contact the OOEL at ESTEC by sending an email to: ooel@esa.int.

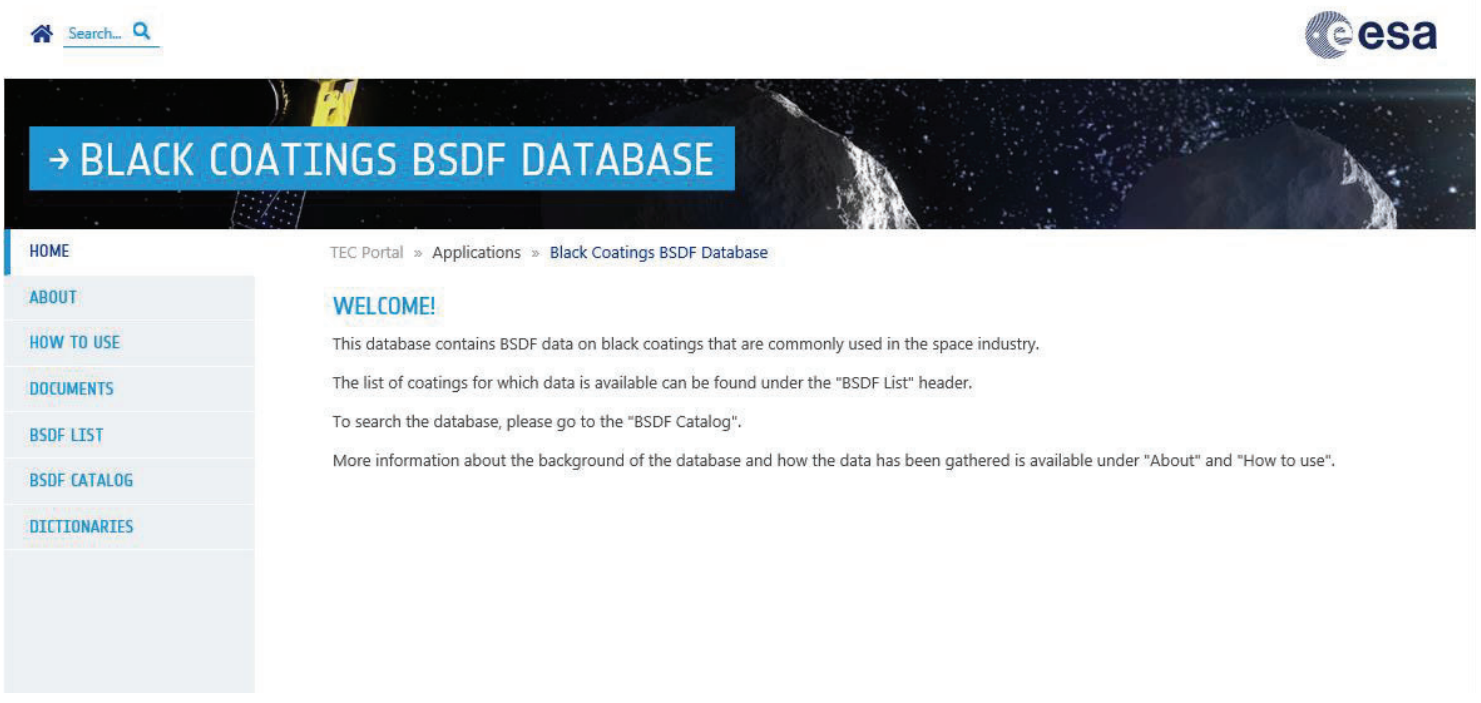

Figure 6: Database home page

The database has been created within the ESA sharepoint environment SPICE. The list of data can be accessed using the tabs on the left side of the screen (see Figure 6). The "About" and "How to use" tabs contain some practical information about the database, most of which is also described in this paper. The tab "BSDF list" contains the full list of data (see Figure 7). Under the tab "BSDF catalog" an advanced search option is available. The BSDF of a coating depends on many factors. As such, each entry in the database is structured according to:

- Coating Type: The name of the coating.

- Coating Info: Any specific information on the coating that could influence the BSDF, if available. For example, coating thickness or way of application.

- Substrate Info: Any specific information on the substrate that could influence the BSDF, if available. For example, substrate material or surface roughness.

- Wavelength: The wavelength at which the BSDF was measured.

- Polarization: Any specific polarization information on the measurement data.

- Measurement Environment: The temperature and humidity environment in which the measurements were performed.

- Measurement Facility: The facility, institution or company at which the BSDF data was measured.

- Measurement date: The date on which the measurement was performed 


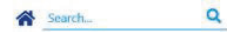

a

esa

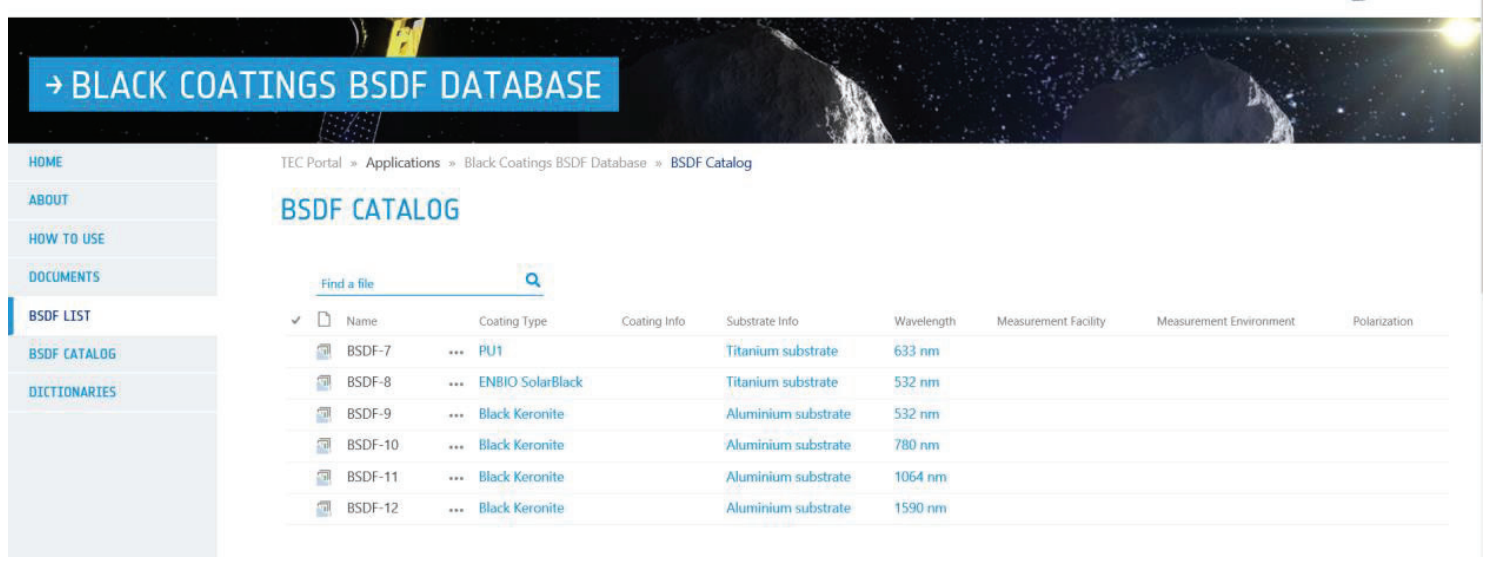

Figure 7: The list of BSDF data

The data for each entry can be accessed by clicking on the name of the entry, which has the form BSDF-\# (with \# being the number of the entry).

When an entry is opened a new list appears of all the files available for that specific entry (see Figure 8). Each file can be opened by clicking on the name of that file. To download a file, select the files to be downloaded, open the "More" drop-down menu that appears on top of the list and click "download". Alternatively, the dropdown menu can also be opened by simply right-clicking on the file to be downloaded.

$$
\text { 소 Search.. Q }
$$

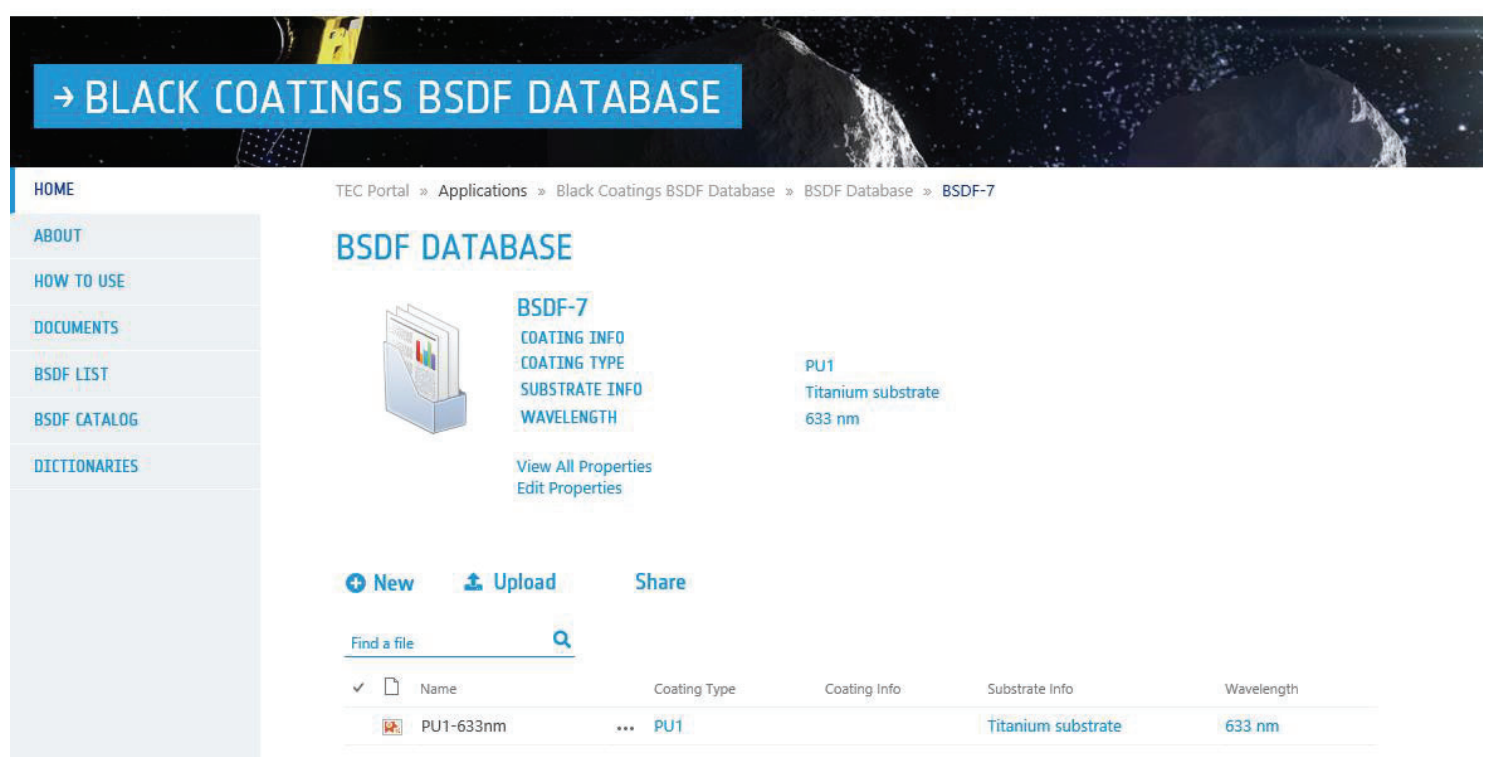

Figure 8: Example of database entry 


\section{CONCLUSION}

In this paper the Black Coatings BSDF Database has been introduced to the space community. The added value of the database has been explained and a short introduction to the functionalities of the database has been presented. This database has as purpose to serve the space community by giving easy access to BSDF data of black coatings. The data in this database can be used for preliminary straylight analysis of black coatings. However, as many factors can influence the BSDF it is still recommended to perform dedicated BSDF measurements when a more detailed straylight analysis is needed to assess the compliance of an instrument to its stringent straylight requirements.

The database is currently in an early development stage and will be continuously updated and improved. In order to make it a complete database, the European space community is invited to cooperate to help populate the database. Cooperation can be done by providing samples for measurements in the OOEL facilities or providing measurement data that can be included into the database. For access, suggestions and cooperation please contact the OOEL by sending an email to ooel@esa.int.

\section{REFERENCES}

[1] J. C. Stover, Optical Scattering: Measurement and Analysis, SPIE Optical Engineering Press, 1995.

[2] D. R. Cheever, F. M. Cad, K. A. Klicker and J. C. Stover, "Design review of a unique complete angle scatter instrument (CASU)," in Proceedings Volume 0818, Current Developments in Optical Engineering II, 1987.

[3] A. von Finck, M. Hauptvogel and A. Duparré, "Instrument for close-to-process light scatter measurements of thin film coatings and substrates," Applied Optics, 2011.

[4] P. Jugniot, M. Nowak, S. Gomes, O. Guillaumon and G. Sierra, "Measurement of solar absorptance of MAP thermal-control coatings," 3108 2018. [Online]. Available: https://www.mapcoatings.com/documents/2007/measurement_of_solar_absorptance_of_map_thermalcontrol_coatings_08_11_2018.pdf.

[5] ENBIO Ltd., "SolarBlack Spec," 2018. [Online]. Available: http://enbio.eu/wpcontent/uploads/2018/10/SolarBlack.pdf. 